Olle KOTTA

\title{
DETERMINATION OF THE STATE-SPACE MODEL OF 2-D SYSTEM FROM ITS INPUT-OUTPUT REPRESENTATION
}

\author{
(Presented by N. Alumäe)
}

\section{Introduction}

In recent years several authors have discussed the two-dimensional (2-D) realization problem of the 2-D transfer function by Roesser's state space model. For a general 2-D transfer function, at this moment there is no technique available for constructing realizations with minimal dimension $\left[{ }^{1}\right]$. In $\left[{ }^{2-4}\right]$ the procedures are given which obtain state space models with low order. For the important special case of 2-D systems with separable denominator transfer function, Sontag $\left[{ }^{4}\right]$ presented an algorithm for obtaining the realization with minimal dimension.

In this paper we also consider the minimal realization problem of separable denominator transfer function by Roesser's model. Unlike $\left[{ }^{4}\right]$, an algorithm is proposed that yields a state space model in the canonical form. The algorithm relies on the fact that the parameters of the transfer function and these of the state space model in the canonical form are connected in a simple way (this has been shown in the paper). From the parametric standpoint the denominator of the transfer function and the matrices $F^{1}$ and $F^{4}$ in the Roesser's model are equivalent. Also, the vectors $h^{1}$ and $h^{2}$ can be explicitly given (they contain only zeros and ones). To obtain the matrix $F^{3}$ and vectors $g^{1}, g^{2}$, three linear equations must be formed and solved. Therefore, in addition to yielding a canonical form realization, the proposed procedure has the advantage of requiring a relatively small amount of computation.

\section{Statement of the problem}

Consider the 2-D LSI system with separable denominator transfer function. The state space model of such system consists of equations

$$
\begin{gathered}
x^{h}(i+1, j)=F^{1} x^{h}(i, j)+g^{1} u(i, j), \\
x^{v}(i, j+1)=F^{3} x^{h}(i, j)+F^{4} x^{v}(i, j)+g^{2} u(i, j), \\
y(i ; j)=h^{1} x^{h}(i, j)+h^{2} x^{v}(i, j), \quad i, j \geqslant 0,
\end{gathered}
$$

where $x^{h}(i, j)=\left[x_{k}^{h}(i, j)\right]$ is an $n \times 1$ horizontal state vector, $x^{v}(i, j)=$ $=\left[x_{k}^{v}(i, j)\right]$ is an $m \times 1$ vertical state vector, $u(i, j)$ is a scalar input, $y(i, j)$ is a scalar output, and $F^{1}, F^{3}, F^{4}, g^{1}, g^{2}, h^{1}, h^{2}$ are real matrices of proper dimensions. The polynomial input-output model of such system is the following:

$$
A_{1}(z) A_{2}(w) y(i, j)=B(z, w) u(i, j)
$$


In $(4), B(z, w)$ is a polynomial in two variables - horizontal forward shift operator $z$ and vertical forward shift operator $w$ :

$$
\begin{gathered}
z u(i, j)=u(i+1, j), \quad w u(i, j)=u(i, j+1), \\
B(z, w)=\sum_{\substack{k=0 \\
k+l \neq N+M}}^{N} \sum_{l=0}^{M} b_{k l} z^{k} w^{l} .
\end{gathered}
$$

$A_{1}(z)$ and $A_{2}(w)$ are polynomials in $z$ and $w$, respectively,

$$
A_{1}(z)=\sum_{k=0}^{N} a_{k}^{1} z^{k}, \quad A_{2}(w)=\sum_{l=0}^{M} a_{N}^{2} w^{l}, \quad a_{N}^{1}=a_{M}^{2}=1
$$

Our purpose here will be to find a link between descriptions (1)-(3) and (4) under the assumptions that

$$
\begin{aligned}
& \operatorname{rank}\left[\left(h^{1}\right)^{T},\left(h^{1} F^{1}\right)^{T} ; \ldots,\left(h^{1}\left(F^{1}\right)^{n-1}\right)^{T}\right]=n, \\
& \operatorname{rank}\left[\left(h^{2}\right)^{T},\left(h^{2} F^{4}\right)^{T}, \ldots,\left(h^{2}\left(F^{4}\right)^{m-1}\right)^{T}\right]=m .
\end{aligned}
$$

The assumption (5a) is somewhat more strict than the corresponding assumption of local observability:

$$
\operatorname{rank}\left[H_{m}^{T},\left(H_{m} F^{1}\right)^{T}, \ldots,\left(H_{m}\left(F^{1}\right)^{n-1}\right)^{T}\right]=n,
$$

where $H_{m}^{T}=\left[\left(h^{1}\right)^{T},\left(h^{2} F^{3}\right)^{T},\left(h^{2} F^{4} F^{3}\right)^{T}, \ldots,\left(h^{2}\left(F^{4}\right)^{m-1} F^{3}\right)^{T}\right]$.

\section{Main result}

As shown in $\left[{ }^{5}\right]$, the 2-D LSI locally observable system under the assumption (5a) can be transformed into the following canonical form

$$
\begin{aligned}
& \underset{n \times n}{F^{1}}=\left[\begin{array}{cc}
0 & I_{n-1} \\
f_{1}^{1} & f_{1}{ }_{n}
\end{array}\right] \quad \underset{m \times m}{F^{4}}=\left[\begin{array}{ccc}
0 & & I_{m-1} \\
f_{4}^{4} & \ldots & f_{m}^{4}
\end{array}\right] \text {, } \\
& \underset{1 \times n}{h^{1}}=\left[\begin{array}{llll}
1 & 0 & \ldots & 0
\end{array}\right], \quad \underset{1 \times m}{h^{2}}=\left[\begin{array}{llll}
1 & 0 & \ldots & 0
\end{array}\right] .
\end{aligned}
$$

The matrices $F^{3}=\left[f_{k s}^{3}\right], g^{1}=\left[g_{k}{ }^{1}\right]$ and $g^{2}=\left[g_{k}{ }^{2}\right]$ have no special structure in this canonical form.

Making use of the canonical structure of the matrices $F^{1}, F^{4}, h^{1}, h^{2}$, it is easy to derive from equations (1)-(3) the following expressions:

$$
\begin{gathered}
x^{h}(i, j)=V(z) y(i, j)-W_{1} V(z) u(i, j)-V(z) x_{1}^{v}(i, j), \\
x^{v}(i, j)=V(w) y(i, j)-W_{2} V(w) u(i, j)-V(w) x_{1}^{h}(i, j)- \\
-\sum_{s=1}^{n} F_{s} V(w) x_{s}^{h}(i, j),
\end{gathered}
$$

where $V(z)=\left[1, z, \ldots, z^{n-1}\right]^{\mathrm{T}}, \quad V(w)=\left[1, w, \ldots, w^{m-1}\right]^{\mathrm{T}}$,

$$
\begin{gathered}
W_{1}=\sum_{k=1}^{n-1} g_{k}^{1} S_{n}^{k}, \quad W_{2}=\sum_{k=1}^{m-1} g_{k}^{2} S_{m}^{k}, \\
F_{s}=\sum_{k=1}^{m-1} f_{k s}^{3} S_{m}^{k},-\quad S_{n \times n}=\left[s_{i j}\right], \quad s_{i j}=\delta_{i, j+1} .
\end{gathered}
$$

The substitution of (6) into (1) yields 


$$
\begin{gathered}
{\left[z I_{n}-F^{1}\right] V(z) y(i, j)=\left\{\left[z I_{n}-F^{1}\right] W_{1} V(z)+g^{1}\right\} u(i, j)+} \\
+\left[z I_{n}-F^{1}\right] V(z) x_{1}^{v}(i, j),
\end{gathered}
$$

and the substitution of (7) into (2) yields

$$
\begin{gathered}
{\left[w I_{m}-F^{\left.4^{*}\right]} V(w) y(i, j)=\left\{\left[w I_{m}-F^{\left.4^{*}\right]} W_{2} V(w)+g^{2}\right\} u(i, j)+\right.\right.} \\
+\left[w I_{m}-F^{4^{*}}\right] V(w) x_{1}^{h}(i, j)+\left[w I_{m}-F^{4^{*}}\right] \sum_{s=1}^{n} F_{s} V(w) x_{s}^{h}(i, j)+ \\
+F^{* * *} x_{1}^{v}(i ; j)+F^{3} x^{h}(i, j),
\end{gathered}
$$

where $\quad F^{4 *}=\left[\begin{array}{cccc}0 & & I_{m-1} \\ 0 & f_{2}^{4} & \ldots & f_{m}^{4}\end{array}\right], \quad F^{4 * *}=\left[\begin{array}{c}0 \\ \vdots \\ 0 \\ f_{1}^{4}\end{array}\right]$

In the system of $n$ equations (8), however, only the $n$-th equation is significant, the remaining ones are simple identities. Similarly, in (9), only the $m$-th equation is significant. Defining $f^{1}{ }_{n+1}=f^{4} m+1=-1$, we have from the $m$-th equation of (9)

$$
\begin{gathered}
x_{1}^{v}(i, j)=1 / f_{1}^{4}\left\{\sum_{l=1}^{m} f_{l+1}^{4} w^{l}\left[x_{1}^{h}(i, j)-y(i, j)\right]+\right. \\
\left.+\sum_{l=0}^{m-1} \sum_{r=1}^{m-l} f_{l+r+1}^{4}\left[g_{r}^{2} w^{l} u(i, j)+\sum_{s=1}^{n} f_{r s}^{3} w^{l} x_{s}^{h}(i, j)\right]\right\} .
\end{gathered}
$$

The substitution of $x_{1}^{v}(i, j)$ into the $n$-th equation of $(8)$ and multiplication by $-f_{1}{ }^{4}$ yields

$$
\begin{gathered}
\sum_{k=0}^{n} \sum_{l=0}^{m} f_{k+1}^{1} f_{l+1}^{4} z^{k} w^{l} y(i, j)-\sum_{k=0}^{n} \sum_{l=0}^{m-1} \sum_{r=1}^{m-l} f_{k+1}^{1} f_{l+r+1}^{4} g_{r}^{2} z^{k} w^{l} u(i, j)- \\
-f_{1}^{4} \sum_{k=0}^{n-1} \sum_{r=1}^{n-k} f_{k+r+1}^{1} g_{r}^{1} z^{k} u(i, j)= \\
=\sum_{t=0}^{n} f_{t+1}^{1} z^{t}\left[\sum_{s=1}^{n} \sum_{l=0}^{m-1} \sum_{r=1}^{m-l} f_{l+r+1}^{4} f_{r s}^{3} w^{l} x_{s}^{h}(i, j)+\sum_{l=1}^{m} f_{l+1}^{4} w^{l} x_{1}^{h}(i, j)\right] .
\end{gathered}
$$

Let us deal with the right hand side of (10). From equation (1) we obtain

$$
\begin{aligned}
z^{n} x_{s}^{h}(i ; j) & =z^{s} x_{n}^{h}(i, j)+\sum_{k=s}^{n-1} g_{k}^{1} z^{n-k+s-1} u(i, j)= \\
& =\sum_{t=0}^{n-1} f_{t+1}^{1} z^{s-1} x_{t}^{h}(i, j)+\sum_{k=s}^{n} g_{k}^{1} z^{n-k+s-1} u(i, j)= \\
& =\sum_{t=0}^{n-1} f_{t+1}^{1} z^{t} x_{s}^{h}(i, j)+\sum_{v=1}^{s-1} \sum_{t=v}^{s-1} f_{v}^{1} g_{t}^{1} z^{v-t+s-2} u(i, j)- \\
& -\sum_{v=s}^{n} \sum_{t=s}^{v} f_{v+1}^{1} g_{t}^{1} z^{v-t+s-1} u(i, j), \quad s=1, \ldots, n
\end{aligned}
$$


which implies that the right hand side of equation (10) may readily be expressed in terms of inputs. Finally, we get:

$$
\begin{aligned}
& \sum_{k=0}^{n} \sum_{l=0}^{m} f_{k+1}^{1} f_{l+1}^{4} z^{k} w^{l} y(i, j)= \\
& =\sum_{k=0}^{n} \sum_{l=0}^{m-1} \sum_{r=1}^{m-l} f_{k+1}^{1} f_{l+r+1}^{4} g_{r}^{2} z^{k} w^{l} u(i, j)+ \\
& \quad+f_{1}^{4} \sum_{k=0}^{n-1} \sum_{r=1}^{n-k} f_{k+r+1}^{1} g_{r}^{1} z^{k} u(i, j)- \\
& \quad-\sum_{s=1}^{n} \sum_{k=0}^{s-2} \sum_{l=0}^{m-1} \sum_{r=1}^{m-l} \sum_{v=0}^{k} f_{l+r+1}^{4} f_{v+1}^{1} g_{v+s-1-k}^{1} f_{r s}^{3} z^{k} w^{l} u(i, j)+ \\
& \quad+\sum_{s=1}^{n} \sum_{k=s-1}^{n-1} \sum_{l=0}^{m-1} \sum_{r=1}^{m-l} \sum_{v=k+1}^{n} f_{l+r+1}^{4} f_{v+1}^{1} g_{v+s-k-1}^{1} f_{r s}^{3} z^{k} w^{l} u(i, j)+ \\
& \quad+\sum_{k=0}^{n-1} \sum_{l=1}^{m} \sum_{r=k+1}^{n} f_{r+1}^{1} f_{l+1}^{4} g_{r-k}^{1} z^{k} w^{l} u(i, j),
\end{aligned}
$$

or more compactly

$$
\sum_{k=0}^{n} \sum_{l=0}^{m} a_{k}^{1} a_{l}^{2} z^{k} w^{l} y(i, j)=\sum_{k=0}^{n} \sum_{l=0}^{m} b_{k l} z^{k} w^{l} u(i, j),
$$

where $a_{k}^{1}=-f_{k+1}^{1}, \quad k=0, \ldots, n, \quad a_{l}^{2} \stackrel{k+l \neq 0}{=}-f_{l+1}^{4}, \quad l=0, \ldots, m$,

$$
\begin{aligned}
& b_{k l}=m_{k l, r s} \cdot f_{r s}^{3}+n_{k l} \text {; }
\end{aligned}
$$

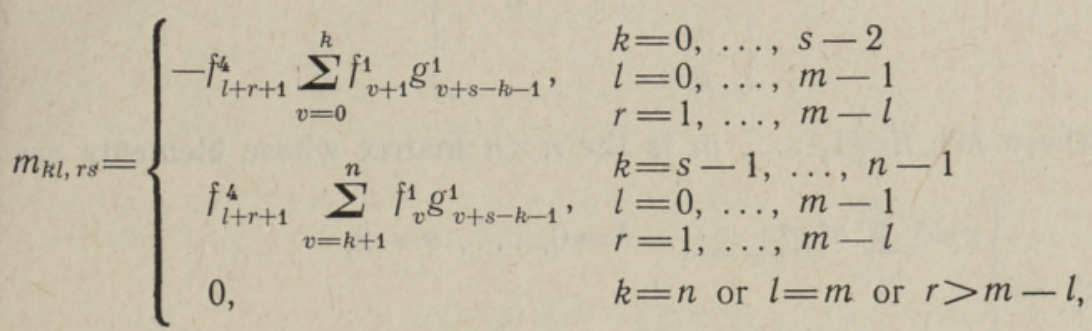

$$
\begin{aligned}
& n_{k l}=f_{k+1}^{1} \sum_{r=1}^{m-1} f_{l+r+1}^{4} g_{r}^{2}+f_{l+1}^{4} \sum_{r=1}^{n-k} f_{k+r+1}^{1} g_{r}^{1} .
\end{aligned}
$$

So, having eliminated the state vectors $x^{h}$ and $x^{v}$ from equations (1)(3), we have reached the polynomial input-output representation.

\section{Algorithm description}

Examining the representation (12) and the system of linear equations (13), one can get the following algorithm for computation of the state space representation from the polynomial input-output representation.

Step 1. $n=N, m=M$.

Step 2. Construct the matrices

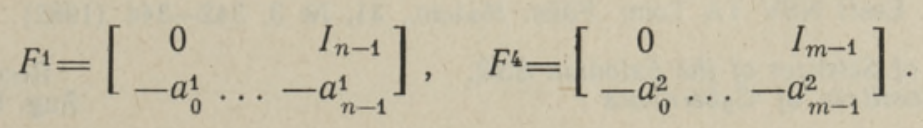


Step 3. Construct the matrices

$$
\begin{aligned}
& N_{1}=\left[\begin{array}{cccc}
a_{1}^{1} & a_{2}^{1} & \ldots & a_{n}^{1} \\
& & \cdot \\
a_{2}^{1} & & \cdot & \\
\vdots & \cdot & 0 \\
\cdot & &
\end{array}\right], \quad \underset{m \times m}{N_{2}}=\left[\begin{array}{cccc}
a_{1}^{2} & a_{2}^{2} & \ldots & a_{m}^{2} \\
a_{n}^{1} & & &
\end{array}\right] \\
& \underset{1 \times n}{b_{1}^{T}}=\left[b_{0 m} b_{1 m} \ldots b_{n-1, m}\right] \text {, } \\
& \underset{1 \times m}{b_{2}^{T}}=\left[b_{n 0} b_{n 1} \ldots b_{n, m-1}\right] .
\end{aligned}
$$

Step 4. $g^{1}=N_{1}^{-1} b_{1}, \quad g^{2}=N_{2}^{-1} b_{2}$.

Note that $N_{1}$ and $N_{2}$ are always nonsingular because of their structure, in fact $\operatorname{det} N_{1}=\operatorname{det} N_{2}=1$.

Step 5. Construct the matrices

$$
\begin{aligned}
& b^{T}=\left[\begin{array}{lllllll}
b_{00} b_{10} & \ldots & b_{n-1,0} & \ldots & b_{0, m-1} b_{1, m-1} & \ldots & b_{n-1, m-1}
\end{array}\right], \\
& n^{T}=\left[\begin{array}{lllllll}
n_{00} n_{10} & \ldots & n_{n-1,0} & \ldots & n_{0, m-1} n_{1, m-1} & \ldots & n_{n-1, m-1}
\end{array}\right], \\
& \underset{n m \times n m}{M}=\left[\begin{array}{cccc}
M^{1} & M^{2} & \ldots & M^{m} \\
M^{2} & & \ddots & \\
\vdots & & \ddots & 0 \\
\vdots & & & \\
M^{m} & & &
\end{array}\right],
\end{aligned}
$$

where $M^{h}, h=1, \ldots, m$ is the $n \times n$ matrix whose elements are

$$
m_{k s}^{h}=\left\{\begin{aligned}
-a_{h}^{2} \sum_{v=0}^{k} a_{v}^{1} g_{v+s-k-1}^{1}, \quad k=0, \ldots, s-2, \\
a_{h}^{2} \sum_{v=h+1}^{n} a_{v}^{1} g_{v+s-k-1}^{1}, \quad k=s-1, \ldots, n-1 .
\end{aligned}\right.
$$

Step 6. $\operatorname{vec}_{r} F^{3}=M^{-1}(b-n)$, where $\operatorname{vec}_{r} F^{3}=\left[f_{11}^{3} \ldots f_{1 n}^{3} \ldots f_{m 1}^{3} \ldots f_{m n}^{3}\right]$. Step 7. Construct the matrix $F^{3}$ from $\operatorname{vec}_{r} F^{3}$.

\section{REFERENCES}

1. Fornasini, E., Marchesini, G. Preprints of 8th IFAC World Congress, 2, $147-153$ 2. Kung, S.-Y., Lévy, B. C., Morf, M., Kailath, T. Proc. IĘEE, 65, № 6, 945-961 (1977)

3. Eising, R. IEEE Trans. Automat. Contr., AC-23, № 10, 793-799 (1978),

4. Sontag, E. D. IEEE Trans. Acoust., Speech and Signal Proc., ASSP-26, № 10 , 480-482 (1978).

5. Kotta, O. Eesti NSV TA Toim. Füüs. Matem., 31, № 3, 342-344 (1982). 


\section{2-D-SUSTEEMI OLEKUMUDELI MAX̆RAMINE SUSTEEMI SISEND-VÄLJUND KIRJELDUSE PÖHJAL}

Tóös on vaadeldud kahedimensioonilise (2-D) eralduva nimetajaga ülekandefunktsiooni $B(z, \omega) / A_{1}(z) A_{2}(\omega)$ realiseerimist minimaalmõôtmelise Roesseri olekumudeli abil:

$$
\begin{aligned}
& x^{h}(i+1, j)=F^{1} x^{h}(i, j)+g^{1} u(i, j), \\
& x^{v}(i, j+1)=F^{3} x^{h}(i, j)+F^{4} x^{v}(i, j)+g^{2} u(i, j), \\
& y(i, j)=h^{1} x^{h}(i, j)+h^{2} x^{v}(i, j) .
\end{aligned}
$$

Erinevalt teadaolevast algoritmist annab töös leitud algoritm olekumudeli kanoonilisel kujul. Algoritm põhineb töös tõestatud lihtsal seosel kanoonilise kujuga olekumudeli ja ülekandefunktsiooni parameetrite vahel.

\section{Юлле КОТTA}

\section{ОПРЕДЕЛЕНИЕ МОДЕЛИ СОСТОЯНИЯ ДВУМЕРНОИ СИСТЕМЫ ПО ЕЕ ВХОД-ВЫХОД ОПИСАНИЮ}

Исследуется проблема минимальной реализации двумерной передаточной функции с отделимым знаменателем $B(z, \omega) / A_{1}(z) A_{2}(\omega)$ с помощью модели состояния Роессера,

$$
\begin{aligned}
& x^{h}(i+1, j)=F^{1} x^{h}(i, j)+g^{1} u(i, j), \\
& x^{v}(i, j+1)=F^{3} x^{h}(i, j)+F^{4} x^{v}(i, j)+g^{2} u(i, j), \\
& y(i, j)=h^{1} x^{h}(i, j)+h^{2} x^{v}(i, j) .
\end{aligned}
$$

Предлагаемый алгоритм в отличие от существующего дает модель состояния в канонической форме. Алгоритм основан на доказанном в статье обстоятельстве, что пар з. метры модели состояния в канонической форме и параметры передаточной функции связаны простым способом. 